Pacific Journal of Mathematic 


\title{
A CERTAIN CLASS OF TOTAL VARIATION MEASURES OF ANALYTIC MEASURES
}

\author{
JUN-ICHI TANAKA
}

In this paper we investigate a problem concerning the total variation measure of an analytic measure induced by a flow. Our main results are: Let $\mu$ be a positive Baire measure on a compact Hausdorff space and let the distant future in $L^{2}(\mu)$ be the zero subspace. If $\mu$ is absolutely continuous with respect to an invariant measure, then $\mu$ is the total variation measure of an analytic measure. On the other hand, if $\mu$ is singular with respect to each invariant measure, then there is a summable Baire function $g$ such that $g d \mu$ is analytic and $g^{-1}$ is bounded. Moreover, we note that general $\mu$ can be uniquely expressed as the sum of measures of above two types.

1. Preliminaries. In this section we establish the notation and terminology which we shall use. We also explain some results about spectra.

Throughout this paper $X$ will denote a fixed compact Hausdorff space upon which the real line $R$ acts as a locally compact transformation group. The translation of $x$ by $t$ in $R$ will be written $x+t$. Let $C(X)$ be the space of all continuous complex-valued functions on $X$. The dual space of $C(X)$ is the space of all bounded complex Baire measure on $X$ and it will be denoted by $M(X)$. For a positive measure $\sigma$ in $M(X),\|\cdot\|_{p}$ will denote the norm in the Lebesgue space $L^{p}(\sigma), 1 \leqq p \leqq \infty$. Let $m$ be a positive invariant measure in $M(X)$. For a function $\phi$ on $X$, we set $\left(T_{t} \phi\right)(x)=\phi(x-t)$. Then the action of $R$ on $X$ induces a strongly continuous one parameter group $\left\{T_{t}\right\}_{t \in R}$ of isometries of $C(X)$ or of $L^{p}(m), 1 \leqq p<\infty$. Notice that $\left\{T_{t}\right\}_{t \in R}$ is merely continuous in the weak-* topology on $L^{\infty}(m)$. Using $\left\{T_{t}\right\}_{t \in R}$ one may convolve a function in $L^{p}(m), 1 \leqq$ $p \leqq \infty$, a function in $C(X)$ and a measure in $M(X)$ with a function in group algebra $L^{1}(R)$ as follows: For $\phi$ in $L^{p}(m)$ or in $C(X)$ and $f$ in $L^{1}(R)$,

$$
\dot{\phi} * f=\int_{R}\left(T_{t} \phi\right) f(t) d t
$$

When $1 \leqq p<\infty$ or when $\phi$ lies in $C(X)$, this integral is a Bochner integral but when $p=\infty$, the integral converges in the weak-* topology. For $\lambda$ in $M(X)$ and $f$ in $L^{1}(R), \lambda * f$ is defined by the equation 


$$
\int \phi d(\lambda * f)=\int \phi * \widetilde{f} d \lambda
$$

for all $\phi$ in $C(X)$ where $\widetilde{f}(t)=f(-t)$. Let $\theta$ be a function in $L^{p}(m)$, $1 \leqq p \leqq \infty$, a function in $C(X)$, or a measure in $M(X)$. We set $J(\theta)=\left\{f \in L^{1}(R) ; \theta * f=0\right\}$. Then $J(\theta)$ is a closed ideal in $L^{1}(R)$. The spectrum of $\theta$ is the hull of the ideal $J(\theta)$ and is denoted by $S p(\theta)$. We shall say that $\theta$ is analytic if $S p(\theta) \subseteq[0, \infty)$. The measure $\nu$ on $R$ will be defined by $d \nu(t)=1 /\left(\pi\left(1+t^{2}\right)\right) d t$ and we shall denote the Lebesgue and Hardy spaces based on $\nu$ by $L^{p}(\nu)$ and $H^{p}(\nu)$, $1 \leqq p \leqq \infty$. Since $m$ is invariant, it follows from Fubini's theorem that if $\phi$ is a function in $L^{p}(m), 1 \leqq p \leqq \infty$, then there is an invariant null set $N$ such that, for $x$ in $X \backslash N$, the function of $t$, $\phi(x+t)$, belongs to $L^{p}(\nu)$ (see [7; Proposition 2.1]). The space of all analytic functions in $C(X)$ will be denoted by $\mathfrak{A}$, which is a uniformly closed subalgebra of $C(X)$ containing the constant functions. For $t$ in $R$, we shall write $C(t, \infty)$ for the space $\{\phi \in C(X) ; S p(\phi) \subseteq(t, \infty)\}$. Let $\mu$ be a positive measure in $M(X)$. Then the distant future in $L^{2}(\mu)$ is defined to be $\bigcap_{-\infty<t<\infty}[C(t, \infty)]_{2}$ where $[C(t, \infty)]_{2}$ is the $L^{2}(\mu)$ closure of $C(t, \infty)$. We refer the reader to [1] for the basic facts about spectra.

In $[2,3]$, Forelli raised the following question in connection with a converse of $\mathrm{F}$. and M. Riesz' theorem:

Let $\mu$ be a positive measure in $M(X)$. If the distant future in $L^{2}(\mu)$ is the zero subspace, is $\mu$ the total variation measure of an analytic measure?

It has been observed by Helson that the answer of this problem is affirmative for some class of measures in the almost periodic setting. More precisely, he showed the following: Let $\mu$ be a positive measure on the quotient of the Bohr group which has the same null sets as Haar measure. If the distant future in $L^{2}(\mu)$ vanishes, then $\mu$ is the total variation measure of an analytic measure $([5,6$; Theorem 23]). This result is not only interesting but also highly important in invariant subspace theory on compact abelian groups (cf. [6] and [4; Ch. VII]).

In our discussion in the forthcoming sections, we frequently use the following theorem of Forelli's concerning the above problem.

Theorem F ([3; Theorem 1]). Let $\mu$ be a positive measure in $M(X)$. Suppose that the distant future in $L^{2}(\mu)$ is the zero subspace. Then there exists a function $g$ in $L^{\infty}(\mu)$ with $0<|g| \leqq 1$ a.e. $\mu$ such that gde is analytic.

The author would like to express his sincere gratitude to Professors Yuji Ito and Junzo Wada for their useful advices. 
2. Absolutely continuous measures. In this section we shall consider a positive measure which is absolutely continuous with respect to an invariant measure. Our result depends on Muhly's work.

THEOREM 2.1. Let $m$ be a positive invariant measure in $M(X)$, and let $w$ be a positive function in $L^{1}(m)$. Suppose that the distant future of $L^{2}(w d m)$ is the zero subspace. Then $w d m$ is the total variation measure of an analytic measure.

In order to prove Theorem 2.1, we need two lemmas and one proposition.

Lemma 2.2. Let $\phi$ be a function in $L^{p}(m), 1 \leqq p \leqq \infty$. Then the following properties are equivarent:

(i) The spectrum of $\dot{\rho}$ as an element of $L^{p}(m)$ is nonnegative:

(ii) The spectrum of $\delta d m$ as a measure in $M(X)$ is nonnegative:

(iii) There exists an invariant null set $N$ such that for any $x$ in $X \backslash N$, the function of $t, \dot{\phi}(x+t)$, belongs to $H^{p}(\nu)$.

This lemma was essentially proved in $[8 ; \S 2]$, so we omit the proof.

We set $\mathfrak{A}_{0}=C(0, \infty)$ (see $\S 1$ for the definition of $C(0, \infty)$ ). Then $\mathfrak{U}_{0}$ is an ideal of $\mathfrak{A}$ by [1; Theorem 1]. For any subset $S$ of $L^{p}(m)$, $1 \leqq p \leqq \infty$, we write:

$$
\begin{aligned}
& \bar{S}=\{\bar{f} ; f \text { is in } S\}, \\
& {[S]_{p}=\text { the } L^{p}(m) \text {-closure of } S .}
\end{aligned}
$$

LEMMA 2.3. There exists a closed subspace $M$ in $L^{2}(m)$ consisting invariant functions such that $L^{2}(m)=\left[\mathfrak{A}_{0}\right]_{2} \oplus M \oplus\left[\overline{\mathscr{Q}}_{0}\right]_{2}$.

Proof. Since $m$ is invariant, we have $S p(m)=\{0\}$ by [7; Proposition 2.2]. So it follows from the preceding remark and [1; Proposition 2] that $\left[\mathfrak{H}_{0}\right]_{2}$ is orthogonal to $\left[\overline{\mathscr{A}_{0}}\right]_{2}$. Let $M$ be the orthogonal complement of $\left[\mathfrak{A}_{0}\right]_{2} \oplus \overline{\left[\mathfrak{R}_{0}\right]_{2}}$ in $L^{2}(m)$. If $\dot{\phi}$ is in $M$, then the measure $\phi d m$ is orthogonal to $\mathfrak{A}_{0}+\overline{\mathfrak{A}}_{0}$. Since $S p(\phi d m) \subseteq\{0\}$ by [1; Proposition 2'], we see that $\phi d m$ is invariant. Hence $\phi$ is an invariant function. This completes the proof.

Proposition 2.4. Suppose that $f$ is a function in $L^{\infty}(m)$ such that $f^{-1}$ is in $L^{\infty}(m)$. Then there exists an analytic function $\phi$ in $L^{\infty}(m)$ such that $|f|=|\dot{\phi}|$ a.e. $m$. 
Proof. By the hypothesis, we see that $\log |f|$ is in $L^{\infty}(m)$. It follows from Lemma 2.3 that $\log |f|=u+v+\bar{u}$ where $u$ is in $\left[\mathfrak{H}_{0}\right]_{2}$ and $v$ is in $M$. Notice that $v$ is a real-valued invariant function in $L^{2}(m)$. If we put $g=2 u+v$, then $g$ is an analytic function in $L^{2}(m)$ by [1; Proposition 2] and Lemma 2.2. Since Re $g=\log |f|$ is bounded, we have that $\exp (g)$ is a bounded function. It follows from Lemma 2.1 and the Fubini's theorem that there is a null set $N$ such that for any $x$ in $X \backslash N, g(x+t)$ belongs to $H^{2}(\nu)$ as a function of $t$ and $\exp (g(x+t))$ belongs to $L^{\infty}(\nu)$ as a function of $t$. The properties of $H^{p}(\nu), 1 \leqq p \leqq \infty$, implies that $\exp (g(x+t))$ belongs to $H^{\infty}(\nu)$ as a function of $t$ for any $x$ in $X \backslash N$. Therefore it follows from Lemma 2.1 that $\exp (g)$ is an analytic function with $|\exp (g)|=|f|$, so the proof is complete.

We are now in a position to prove Theorem 2.1.

Proof of Theorem 2.1. We use the modification argument in the proof of [10; Theorem 1]. It follows from Theorem $F$ in $\S 1$ that there exists a function $\psi$ in $L^{\infty}(m)$ with $0<|\psi(x)| \leqq 1$ a.e. $m$ such that $\psi w d m$ is an analytic measure. We put

$$
H_{n}=\left\{x \in X ;(n+1)^{-1}<|\psi(x)| \leqq n^{-1}\right\}
$$

and define

$$
u_{n}= \begin{cases}|\psi|^{-1} & \text { on } H_{n}, \\ 2^{-(n+1)} & \text { on } X \backslash H_{n}\end{cases}
$$

for $n=1,2, \cdots$. Since both $u_{n}$ and $u_{n}^{-1}$ are in $L^{\infty}(m)$, it follows from Proposition 2.4 that there exists an analytic function $\phi_{n}$ such that $\left|\phi_{n}\right|=u_{n}$. For a.e. $x$ in $H_{m}$, we have

$$
\begin{aligned}
\sum_{n=1}^{\infty}\left|\phi_{n} \psi(x)\right| & =\left(\sum_{n \neq m}\left|\phi_{n} \psi(x)\right|\right)+\left|\phi_{m} \psi(x)\right| \\
& \leqq \sum_{n \neq m} 2^{-(n+1)}+1 \\
& <3 / 2 \\
\left|\sum_{n=1}^{\infty} \phi_{n} \psi(x)\right| & \geqq\left|\phi_{m} \psi(x)\right|-\left(\sum_{n \neq m} \phi_{n} \psi(x)\right) \\
& >1-\sum_{n \neq m} 2^{-(n+1)} \\
& >1 / 2 .
\end{aligned}
$$

Since $m\left(X \backslash \bigcup_{m=1}^{\infty} H_{m}\right)=0$, if we set $g=\sum_{n=1}^{\infty} \phi_{n} \psi$, then both $g$ and $g^{-1}$ are in $L^{\infty}(m)$. From the bounded convergence theorem and [1;

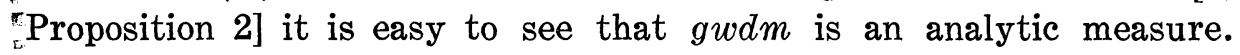
It follows from Proposition 2.4 that there is an analytic function $h$ 
in $L^{\infty}(m)$ such that $|h|=|g|^{-1}$. So $h g w d m$ is an analytic measure whose total variation measure is $w d m$. This completes the proof.

We conclude this section with an application of Theorem 2.1. Recall that the point $x$ in $X$ is said to be a fixed point if $x+t=x$ for any $t$ in $R$, and that the point $x$ in $X$ is said to be a periodic point if $x$ is not a fixed point but $x+t=x$ for some $t$ in $R$. We also recall that a measure in $M(X)$ is said to be quasi-invariant in case every translate of each null set in a null set. What we prove is the following:

Proposition 2.5. Let $\mu$ be a positive measure in $M(X)$ such that the distant future in $L^{2}(\mu)$ is the zero subspace. Suppose that for $\mu$-a.e. $x$ in $X, x$ is a fixed point or a periodic point. Then $\mu$ is the total variation measure of an analytic measure.

Proof. We put

$$
\tau(x)=\inf \{t ; t \text { is positive and } x+t=x\} \text {. }
$$

Since $(t, x) \rightarrow(x+t, x)$ is a continuous function from $[0, \infty) \times X$ to $X \times X$, if we set $S=\{(t, x) ;(x+t, x) \in \Delta\}$ where $\Delta$ is the diagonal set, then $S$ is closed. Notice that

$$
\{x \in X ; 0<\tau(x) \leqq \alpha\}=\{x \in X ;(t, x) \in S \cap(0, \alpha] \times X\} .
$$

Since $(t, x) \rightarrow x$ is continuous, we see that $\tau(x)$ is measurable. Let

$$
\begin{aligned}
& P_{0}=\{x \in X ; x \text { is a fixed point }\}, \text { and } \\
& P_{n}=\{x \in X ; n-1<\tau(x) \leqq n\}
\end{aligned}
$$

for $n=1,2, \cdots$. So $P_{n}$ is an invariant set and $\mu\left(X \backslash \bigcup_{n=0}^{\infty} P_{n}\right)=0$. We set $\mu_{n}(E)=\mu\left(E \cap P_{n}\right)$ for any Baire set $E$, and let $\lambda_{0}(E)=\mu_{0}(E)$ and

$$
\lambda_{j}(E)=\int_{X}\left[\int_{0}^{\tau(x)} \chi_{E}(x+s) d s\right] d \mu_{j}(x)
$$

for $j=1,2, \cdots$. Recall that it follows from [1; Theorem 2] that $\mu$ is quasi-invariant, so $\mu_{n}$ is also quasi-invariant because $P_{n}$ is an invariant set. We can easily observe that $\mu_{n}$ is absolutely continuous with respect to $\lambda_{n}$. For any $u$ in $R$,

$$
\begin{aligned}
\lambda_{n}(E-u) & =\int_{X}\left[\int_{0}^{\tau(x)} \chi_{E-u}(x+s) d s\right] d \mu_{n}(x) \\
& =\int_{X}\left[\int_{0}^{\tau(x)} \chi_{E}(x+s+u) d s\right] d \mu_{n}(x)
\end{aligned}
$$




$$
\begin{aligned}
& =\int_{X}\left[\int_{u}^{\tau(x)+u} \chi_{E}(x+s) d s\right] d \mu_{n}(x) \\
& =\int_{X}\left[\int_{u}^{\tau(x)} \chi_{E}(x+s) d s+\int_{\tau(x)}^{\tau(x)+u} \chi_{E}(x+s) d s\right] d \mu_{n}(x) \\
& =\int_{X}\left[\int_{0}^{\tau(x)} \chi_{E}(x+s) d s\right] d \mu_{n}(x) \\
& =\lambda_{n}(E) .
\end{aligned}
$$

Therefore $\lambda_{n}$ is an invariant measure in $M(X)$. We set

$$
\lambda(E)=\sum_{n=0}^{\infty} 2^{-n}|| \lambda_{n} \|^{-1} \lambda_{n}(E) .
$$

Then $\lambda$ is an invariant measure in $M(X)$ and $\mu$ is absolutely continuous with respect to $\lambda$. From this fact and Theorem 2.1, we have that $\mu$ is the total variation measure of an analytic measure. This completes the proof.

3. Decomposition of quasi-invariant measures. In this section, we would like to add some propositions which follow immediately by virtue of elementary results of spectra. The proof of the following proposition is a straightforward applications of [7; Proposition 2.2] and [1; Proposition 2], so it will not be given.

Proposition 3.1. Let $\mu$ be a measure in $M(X)$. Then $\mu$ is invariant if and only if $\mu$ is orthogonal to $\mathfrak{A}_{0}+\overline{\mathfrak{A}}_{0}$, i.e., for any $f$ and $g$ in $\mathfrak{A}_{0}, \int(f+\bar{g}) d \mu=0$.

Although the following proposition is similar to Muhly's results ([9; Lemma 5.3 and Corollary 5.4]), his proof does not work directly in our case. So we give here a complete proof.

Proposition 3.2. Let $\mu$ be a quasi-invariant measure in $M(X)$. Then $\mu$ is singular with respect to each invariant measure in $M(X)$ if and only if $\mathfrak{A}_{0}+\overline{\mathfrak{A}}_{0}$ is weak-* dense in $L^{\infty}(|\mu|)$.

Proof. Suppose that there is an invariant measure $m$ in $M(X)$ such that $\mu$ is not singular with respect to $m$. Then we have $m=$ $g d|\mu|+\lambda$ where $g$ is an nonnull function in $L^{1}(|\mu|)$ and $\lambda$ is singular with respect to $|\mu|$. Since $\mu$ is quasi-invariant, it follows from [1; Lemma 6] that $g d|\mu|$ is an invariant measure. So $g$ is orthogonal to $\mathfrak{A}_{0}+\overline{\mathfrak{A}}_{0}$ in $L^{1}(|\mu|)$ by Proposition 3.1. Hence $\mathfrak{A}_{0}+\overline{\mathfrak{A}}_{0}$ is not weak-* dense in $L^{\infty}(|\mu|)$. So we have a contradiction. The converse is clear by Proposition 3.1, so the proof is complete. 
We remark that the following corollary is concerned with a problem raised by Helson and Lowdenslager (cf. [9; Remark 5.5]).

CoRollary 3.3 ([9; Corollary 5.4]). Let $\mu$ be a positive measure in $M(X)$. If $\mu$ is singular with respect to each invariant measure, then the subspace $\left\{\bigcap_{s<0}[C[s, \infty)]_{2}\right\} \ominus[C(0, \infty)]_{2}$ consists of the zero function alone, where $[C[s, \infty)]_{2}$ denotes the $L^{2}(\mu)$-closure of $C[s, \infty)$.

Proof. We may assume that the distant future in $L^{2}(\mu)$ is the zero subspace. So, by [1; Theorem 2], $\mu$ is a quasi-invariant measure. Let $f$ be a function in $\left\{\bigcap_{s<0}[C[s, \infty)]_{2}\right\} \ominus[C(0, \infty)]_{2}$. It follows from [1; Theorem 1] that, for any $g$ in $\mathfrak{A}_{0}=C(0, \infty), g f$ lies in $[C(0, \infty)]_{2}$. Then $g f$ is orthogonal to $f$ in $L^{2}(\mu)$. Therefore, since $|f|^{2} d \mu$ is real measure, it is easy to see that $|f|^{2}$ is orthogonal to $\mathfrak{A}_{0}+\overline{\mathfrak{A}}_{0}$ in $L^{1}(\mu)$. So we have that $f=0$ by Proposition 3.2. This completes the proof.

Proposition 3.4. Let $\mu$ be a quasi-invariant measure in $M(X)$. Then $\mu$ is uniquely expressible in the form $\mu=\mu_{1}+\mu_{2}$ where $\mu_{1}$ is mutually absolutely continuous with respect to an invariant measure and $\mu_{2}$ is singular with respect to each invariant measure.

Proof. By above Proposition 3.2, we may assume that $\mathfrak{A}_{0}+\overline{\mathfrak{A}}_{0}$ is not weak-* dense $L^{\infty}(|\mu|)$. We use the Forelli's argument in [2; §2.4]. Let $G$ be a maximal collection of nonnull functions in $L^{1}(|\mu|)$ with the properties that $g d|\mu|$ is orthogonal to $\mathfrak{A}_{0}+\overline{\mathfrak{A}}_{0}$ for all $g$ in $G$ and that if $g$ and $g^{\prime}$ belong to $G$ and $g \neq g^{\prime}$, then $g g^{\prime}=0$. From our assumption, it can be easily seen that $G$ is not empty. Since $\mu$ is finite, $G$ is at most countable, so we can write $G=\left\{g_{1}, g_{2}, \cdots\right\}$. We define

$$
d m(x)=\sum_{n=1}^{\infty} 2^{-n}|| g_{n} \|_{1}^{-1}\left|g_{n}\right| d|\mu|(x)
$$

where $\|\cdot\|_{1}$ denotes the $L^{1}(|\boldsymbol{\mu}|)$-norm. Then $m$ is invariant by Proposition 3.1. Therefore, by the Lebesgue's decomposition theorem, we have $d \mu=h d m+d \lambda$ where $h$ is in $L^{1}(m)$ and $\lambda$ is singular with respect to $m$. Since $G$ is maximal, it is easy to see that $\lambda$ is singular with respect to each invariant measure by Proposition 3.1. The uniqueness of decomposition follows from this fact, so the proof is finished.

REMARK. Let $\mu$ be a positive measure in $M(X)$. By [2; Proposition 2], if we assume that the distant future in $L^{2}(\mu)$ is the zero subspace and that $\mu=\mu_{1}+\mu_{2}$ is the decomposition of Proposition 3.4, then the distant future in $L^{2}\left(\mu_{1}\right)$ and the distant future in $L^{2}\left(\mu_{2}\right)$ are 
both the zero subspaces. Therefore, when we deal with the Forelli's problem, it suffices to consider the case where a measure is singular with respect to each invariant measure.

4. Singular measures. The purpose of this section is to show an improvement of Theorem $\mathrm{F}$ in $\S 1$ under the condition in preceding remark (see §3). We also give an application of this result. In $4.1,4.2,4.4$, and 4.5 , we assume that $\mu$ is a positive measure in $M(X)$ which is singular with respect to each invariant measure. Recall that $\mathfrak{A}_{0}+\overline{\mathfrak{A}}_{0}$ is weak-* dense in $L^{\infty}(\mu)$ if and only if $\mu$ is such a measure (cf. Proposition 3.2).

THEOREM 4.1. Let $1 \leqq p<\infty$. If $\phi$ is a function in $L^{\infty}(\mu)$ with $0<|\phi| \leqq 1$ a.e. $\mu$, then there exists a function $g$ in $\left[\mathfrak{A}_{0} \phi\right]_{p}$ such that $g^{-1}$ is in $L^{\infty}(\mu)$, where $\left[\mathfrak{A}_{0} \phi\right]_{p}$ denotes the $L^{p}(\mu)$-closure of $\mathfrak{A}_{0} \phi$.

In order to prove Theorem 4.1, we provide some lemmas.

LEMMA 4.2. We have the following properties;

(i) If $u$ is a real-valued function in $L^{\infty}(\mu)$, then there exists a sequence $\left\{f_{n}\right\}$ in $\mathfrak{A}_{0}$ such that, for a.e. $x$ in $X, \operatorname{Re} f_{n}(x) \leqq\|u\|_{\infty}$ and $\operatorname{Re} f_{n}(x) \rightarrow u(x)$ (as $\left.n \rightarrow \infty\right)$.

(ii) If $w$ is a positive function in $L^{\infty}(\mu)$ where $w^{-1}$ is in $L^{\infty}(\mu)$, then there exists a sequence $\left\{g_{n}\right\}$ in $\mathfrak{A}_{0}$ such that $\left\|\left|g_{n}\right|-w\right\|_{p} \rightarrow 0$ (as $n \rightarrow \infty)$.

Proof. (i) Let $C_{R}(X)$ be the space of all real-valued continuous functions on $X$. Since there exists a sequence $\left\{u_{n}\right\}$ in $C_{R}(X)$ such that, for a.e. $x$ in $X, u_{n}(x) \leqq\|u\|_{\infty}$ and $u_{n}(x) \rightarrow u(x)$ (as $\left.n \rightarrow \infty\right)$, so we may assume that $u$ is continuous. Let $B$ be the subspace of $C_{R}(X)$ spanned by $\operatorname{Re} \mathfrak{A}_{0}$ and $u$. The functional $L$ can be defined on $B$ by setting:

$$
L(v)=\sup \left\{\int h d \mu ; h \in \operatorname{Re} \mathfrak{A}_{0} \text { and } h \leqq v\right\} .
$$

Then $L$ is a bounded positive linear functional on $B$. We can extend $L$ monotonically no $C_{R}(X)$ (cf. [4; Ch. II §2]). So, by Riesz' representation theorem, there is a positive measure $\sigma$ in $M(X)$ such that

$$
L(v)=\int v d \sigma, \text { for } v \text { in } B .
$$

Since $\sigma-\mu$ is a real measure and orthogonal to $\mathfrak{A}_{0}+\overline{\mathfrak{A}}_{0}$, it follows from Proposition 3.1 that $\sigma-\mu$ is an invariant measure. We put 
$\lambda=\sigma-\mu$, then $\lambda$ is singular with respect to $\mu$ by our assumption. So $\lambda$ is positive. From the definition of linear functional $L$, there exists a sequence $\left\{f_{j}\right\}$ in $\mathscr{A}_{0}$ such that $\operatorname{Re} f_{j}(x) \leqq u(x)$ and $\int\left(u(x)-\operatorname{Re} f_{j}(x)\right) d(\lambda+\mu) \rightarrow 0 \quad$ (as $\left.j \rightarrow \infty\right)$. Therefore we can find a subsequence $\left\{f_{n}\right\}$ of $\left\{f_{j}\right\}$ which has desired properties.

(ii) If we set $u=\log w$, then $u$ is in $L^{\infty}(\mu)$ by the assumption. Therefore we can find sequence $\left\{f_{n}\right\}$ in $\mathfrak{A}_{0}$ which has the properties of (i). Let $g_{n}=\exp \left(f_{n}\right)$. Then, it follows from the bounded convergence theorem that $\left\|\left|g_{n}\right|-w\right\|_{p} \rightarrow 0($ as $n \rightarrow \infty)$. So the proof is finished.

The following result is the well-known Borel-Cantelli lemma, so we omit the proof.

Lemma 4.3. Let $A_{n}$ be a sequence of measurable sets. If $\sum_{n=1}^{\infty} \mu\left(A_{n}^{c}\right)<\infty$, then we have $\mu\left(\overline{\lim }_{n \rightarrow \infty} A_{n}^{c}\right)=0$.

Proof of Theorem 4.1. We first note that if we set

$$
H_{k}=\left\{x \in X ; k^{-1}<|\phi(x)| \leqq 1 \text { a.e. } \mu\right\}
$$

for $k=2,3, \cdots$, then $\mu\left(\bigcup_{k=2}^{\infty} H_{k}\right)=\mu(X)$. By induction, we can choose a sequence $\left\{f_{i}\right\}$ in $\mathfrak{A}_{0}$ and a increasing sequence $\left\{j_{i}\right\}$ of positive integers which have the following properties:

(i) If we set

$$
A_{n}=\left\{x \in X ;\left|\sum_{i=1}^{n} f_{i} \phi(x)\right|>2^{-1}\right\},
$$

then $\mu\left(A_{n}^{c}\right)<2^{-j_{n}}$.

$$
\left\|f_{n} \phi\right\|_{p}<\left(2^{-p^{-1}}\right)^{j_{n-1}}+2^{-n}
$$

Indeed, for some $k_{1}$, we have $\mu\left(H_{k_{1}}\right)>2^{-1}$. From (ii) of Lemma 4.2, we can easily find a function $f_{1}$ in $\mathfrak{A}_{0}$ such that $\mu\left(A_{1}\right)>2^{-1}$ and $\left\|f_{1} \phi\right\|_{p}<2^{-p^{-1}}$. We let $j_{1}=1$. Next, assume by induction that we have found $\left\{f_{1}, f_{2}, \cdots, f_{n}\right\}$ in $\mathfrak{A}_{0}$ and $\left\{j_{1}, j_{2}, \cdots, j_{n-1}\right\}$ which have above properties. It suffices to consider the case where $\mu\left(A_{n-1}^{c}\right)>0$. Let $j_{n}$ and $k_{n}$ be positive integers such that $\mu\left(A_{n-1}^{c}\right)>2^{-j_{n}}$ and $\mu\left(\left(A_{n-1} U\right.\right.$ $\left.\left.H_{k_{n}}\right)^{c}\right)<2^{-j_{n}}$ respectively. We put $E_{n}=H_{k_{n}} \backslash A_{n-1}$ and $b=2^{-j_{n}}-$ $\mu\left(\left(A_{n-1} \cup H_{k_{n}}\right)^{c}\right)$. It follows from Lusin's theorem that there exists a compact subset $K_{n}$ of $A_{n-1}$ such that $\phi$ is continuous on $K_{n}$ and $\mu\left(A_{n-1} \backslash K_{n}\right)<3^{-1} b$. Since $\sum_{i=1}^{n-1} f_{i} \phi$ is continuous on $K_{n}$, if we put $a=\min \left\{\left|\sum_{i=1}^{n-1} f_{i} \phi(x)\right| ; x \in K_{n}\right\}$, then $a>2^{-1}$. Define

$$
u_{n}(x)=\left\{\begin{array}{lll}
1+2^{-n} & \text { on } & E_{n} \\
\min \left(3^{-1}\left(a-2^{-1}\right), 2^{-n}\right) & \text { on } & E_{n}^{c}
\end{array} .\right.
$$


Then it follows from (ii) of Lemma 4.2 that there exist a function $f_{n}$ in $\mathfrak{Q}_{0}$ and a measurable set $E$ with $\mu(E)<3^{-1} b$ such that $\left|f_{n} \phi\right|>$ $1+2^{-(n+1)}$ on $E_{n} \backslash E, \quad\left|f_{n} \phi\right|<3^{-1}\left(a-2^{-1}\right)$ on $E_{n}^{c} \backslash E$, and $\left\|f_{n} \phi\right\|_{p}<$ $\mu\left(E_{n}\right)^{p^{-1}}+2^{-n}$. Then we have $\left(E_{n} \backslash E\right) \cup\left(K_{n} \backslash E\right) \subseteq A_{n}$. In fact, we see that

$$
\begin{aligned}
\left|\sum_{i=1}^{n} f_{i} \phi\right| & \geqq\left|f_{n} \phi\right|-\left|\sum_{i=1}^{n-1} f_{i} \phi\right| \\
& >\left(1+2^{-(n+1)}\right)-2^{-1} \\
& >2^{-1} \text { on } E_{n} \backslash E
\end{aligned}
$$

and

$$
\begin{aligned}
\left|\sum_{i=1}^{n} f_{i} \phi\right| & \geqq\left|\sum_{i=1}^{n-1} f_{i} \phi\right|-\left|f_{n} \phi\right| \\
& >a-3^{-1}\left(a-2^{-1}\right) \\
& >2^{-1} \text { on } K_{n} \backslash E
\end{aligned}
$$

On the other hand, since

$$
\sum_{n=0}^{\infty}\left\|f_{n} \phi\right\|_{p} \leqq \sum_{n=1}^{\infty}\left\{\left(2^{-p^{-1}}\right)^{j_{n-1}}+2^{-n}\right\},
$$

we see that $g=\sum_{n=1}^{\infty} f_{n} \phi$ belongs to $\left[\mathfrak{A}_{0} \phi\right]_{p}$. It follows from Lemma 4.3 that $|g(x)| \geqq 2^{-1}$ a.e. $\mu$, so $g^{-1}$ is in $L^{\infty}(\mu)$. This completes the proof.

REMARK. It is easy to see that if $|\phi|$ is continuous, more generally lower semi-continuous, then we can choose $g$ in $\left[\mathfrak{H}_{0} \phi\right]_{p}$ such that both $g$ and $g^{-1}$ are in $L^{\infty}(\mu)$.

From Theorem 4.1, we have the following improvement of Theorem $\mathrm{F}$ in $\S 1$.

COROLLARY 4.4. If the distant future in $L^{2}(\mu)$ is the zero subspace, then there exists a function $g$ in $L^{p}(\mu), 1 \leqq p<\infty$, such that gde is analytic and $g^{-1}$ is in $L^{\infty}(\mu)$.

Proof. By Theorem F, there is a function $\phi$ in $L^{\infty}(\mu)$ such that $0<|\phi| \leqq 1$ a.e. $\mu$ and $\phi d \mu$ is analytic. Moreover, for any $f$ in $\mathfrak{A}$, we see that $f \phi d \mu$ is analytic by [1; Proposition 2]. From this fact and Theorem 4.1, we find a function $g$ in $L^{p}(\mu)$ which has the desired properties. So the proof is finished.

Finally we give an application of Theorem 4.1 that is an extention of one result in [5]. For a positive measure $\sigma$ in $M(X)$, we 
call that $\sigma$ has the property $(P)$ if $\sigma$ satisfies the following condition:

(P) If $f$ is a function in $\mathfrak{A}$, then there exists an invariant function $\phi_{f}$ in $[\overline{\mathfrak{A}}]_{2}$ such that $f+\phi_{f}$ is orthogonal to $\mathfrak{A}$.

Proposition 4.5. Let $\mu$ be a positive measure in $M(X)$ which satisfies the above condition (P). Suppose that $w$ is a positive function in $L^{2}(\mu)$ such that the distant future in $L^{2}(w d \mu)$ is the zero subspace. Then wde is the total variation measure of an analytic measure.

Proof. Since $w d \mu$ is mutually absolutely continuous with respect to $\mu$, it follows from Theorem $\mathrm{F}$ that there exists a function $\phi$ in $L^{\infty}(\mu)$ such that $0<|\phi| \leqq 1$ a.e. $\mu$ and $\phi w d \mu$ is analytic. From Theorem 4.1, we can find a function $g$ in $\left[\mathfrak{H}_{0} \phi\right]_{2}$ such that $g^{-1}$ is in $L^{\infty}(\mu)$. Notice that $-\log |g|$ is in $L^{2}(\mu)$ and upper bounded. Let $c$ be a positive number with $-\log |g| \leqq c$ a.e. $\mu$. Then it follows from (i) of Lemma 4.2 that there exists a sequence $\left\{f_{n}\right\}$ in $\mathfrak{A}_{0}$ such that $\operatorname{Re} f_{n} \leqq c$ a.e. and $\left\|\operatorname{Re} f_{n}-(-\log |g|)\right\|_{2} \rightarrow 0 \quad$ (as $\left.n \rightarrow \infty\right)$. On the other hand, for any $f$ in $\mathfrak{A}$, we let $\phi_{f}$ be the function as in the condition $(\mathrm{P})$. Then, since $\bar{\phi}_{f}$ is in $[\mathfrak{H}]_{2}$, we have:

$$
\begin{aligned}
4\|\operatorname{Re} f\|_{2}^{2} & =\|f+\bar{f}\|_{2}^{2} \\
& =\left\|\left(f+\phi_{f}\right)+\left(\bar{f}-\phi_{f}\right)\right\|_{2}^{2} \\
& \geqq\left\|f+\phi_{f}\right\|_{2}^{2}, \text { and }\left\|f-\bar{\phi}_{f}\right\|_{2}^{2} .
\end{aligned}
$$

Let $\phi_{n}$ be the invariant function corresponding to $f_{n}$. Then, since $\left\{\operatorname{Re} f_{n}\right\}$ is a Cauchy sequence in $L^{2}(\mu)$, both sequences $\left\{f_{n}+\phi_{n}\right\}$ and $\left\{f_{n}-\bar{\phi}_{n}\right\}$ are also Cauchy sequences. So $\left\{f_{n}+i \operatorname{Im} \phi_{n}\right\}$ is a Cauchy sequence. Hence we can choose $h$ in $L^{2}(\mu)$ such that Re $h=-\log |g|$ a.e. $\mu$ and

$$
\left.\left(f_{n}+i \operatorname{Im} \phi_{n}\right)(x) \longrightarrow h(x) \text { a.e. (as } n \longrightarrow \infty\right) \text {. }
$$

Notice that if $\lambda$ is an analytic measure and if $\psi$ is an invariant function in $L^{1}(|\lambda|)$, then $\psi d \lambda$ is analytic. So it can be easily seen that $\exp \left(f_{n}+i \operatorname{Im} \phi_{n}\right) g w d \mu$ is analytic for $n=1,2, \cdots$. Since

$$
\left|\exp \left(f_{n}+i \operatorname{Im} \phi_{n}\right) g w\right| \leqq e^{c}|g| w
$$

and $|g| w$ is in $L^{1}(\mu)$, it follows from bounded convergence theorem that $\exp (h) g w d \mu$ is analytic. Since $|\exp (h)|=|g|^{-1}$, we see that $w d \mu$ is the total variation measure of the analytic measure $\exp (h) g w d \mu$, so the proof is complete. 


\section{REFERENCES}

1. F. Forelli, Analytic and quasi-invariant measures, Acta Math., 118 (1967), 33-59.

2. - What makes a positive measure the total variation measure of an analytic measure? J. London Math. Soc., (2), 2 (1970), 713-718.

3. - Fourier theory and flows, Actes, Congrès intern. Math., 2 (1970), 456-.457

4. T. Gamelin, Uniform Algebras, Prentice-Hall, Englewood Cliffs, N.J, 1969.

5. H. Helson, Compact groups with ordered duals IV, Bull. London Math. Soc., 5 (1973), 67-69.

6. - Analyticity on compact abelian groups, Algebras in Analysis, Academic Press, (1975), 1-62.

7. P. Muhly, Function algebras and fiows I, Acta Sci. Math. (Szeged), 35 (1973), 111-121.

8. — Founction algebras and flows III, Math. Z., 136 (1974), 253-260.

9. _ The distant future, Indiana Math. J., 24 (1974), 149-159.

10. J. Tanaka, Some remarks on simply invariant subspaces on compact abelian groups, J. Math. Soc. Japan, 30 (1978), 475-482.

11. — A note on Helson's existence theorem, Proc. Amer. Math. Soc., 69(1978), $87-90$.

Received April 13, 1978.

TSURU UNIVERSITY

Tsuru City, Yamanashi-Ken

402 JAPAN 


\section{PACIFIC JOURNAL OF MATHEMATICS}

\section{EDITORS}

Donald BABBITT (Managing Editor)

University of California

Los Angeles, CA 90024

HUGo RossI

University of Utah

Salt Lake City, UT 84112

C. C. MOORE and ANDREW OGG

University of California

Berkeley, CA 94720

\section{J. DuGUNDJI}

Department of Mathematics

University of Southern California

Los Angeles, CA 90007

R. FINN and J. Milgram

Stanford University

Stanford, CA 94305

ASSOCIATE EDITORS
E. F. BECKENBACH
B. H. NeumanN
F. WOLF
K. YoSHIDA

\section{SUPPORTING INSTITUTIONS}

\author{
UNIVERSITY OF BRITISH COLUMBIA \\ CALIFORNIA INSTITUTE OF TECHNOLOGY \\ UNIVERSITY OF CALIFORNIA \\ MONTANA STATE UNIVERSITY \\ UNIVERSITY OF NEVADA, RENO \\ NEW MEXICO STATE UNIVERSITY \\ OREGON STATE UNIVERSITY \\ UNIVERSITY OF OREGON
}

\author{
UNIVERSITY OF SOUTHERN CALIFORNIA \\ STANFORD UNIVERSITY \\ UNIVERSITY OF HAWAII \\ UNIVERSITY OF TOKYO \\ UNIVERSITY OF UTAH \\ WASHINGTON STATE UNIVERSITY \\ UNIVERSITY OF WASHINGTON
}

The Supporting Institutions listed above contribute to the cost of publication of this Journal, but they are not owners or publishers and have no responsibility for its content or policies.

Mathematical papers intended for publication in the Pacific Journal of Mathematics should be in typed form or offset-reproduced, (not dittoed), double spaced with large margins. Please do not use built up fractions in the text of the manuscript. However, you may use them in the displayed equations. Underline Greek letters in red, German in green, and script in blue. The first paragraph or two must be capable of being used separately as a synopsis of the entire paper. Please propose a heading for the odd numbered pages of less than 35 characters. Manuscripts, in triplicate, may be sent to any one of the editors. Please classify according to the scheme of Math. Reviews, Index to Vol. 39. Supply name and address of author to whom proofs should be sent. All other communications should be addressed to the managing editor, or Elaine Barth, University of California, Los Angeles, California, 90024.

50 reprints to each author are provided free for each article, only if page charges have been substantially paid. Additional copies may be obtained at cost in multiples of 50 .

The Pacific Journal of Mathematics is issued monthly as of January 1966. Regular subscription rate: $\$ 84.00$ a year (6 Vols., 12 issues). Special rate: $\$ 42.00$ a year to individual members of supporting institutions.

Subscriptions, orders for numbers issued in the last three calendar years, and changes of address should be sent to Pacific Journal of Mathematics, P.O. Box 969, Carmel Valley, CA 93924, U.S.A. Older back numbers obtainable from Kraus Periodicals Co., Route 100, Millwood, NY 10546.

PUBLISHED BY PACIFIC JOURNAL OF MATHEMATICS, A NON-PROFIT CORPORATION

Printed at Kokusai Bunken Insatsusha (International Academic Printing Co., Ltd.). 8-8, 3-chome, Takadanobaba, Shinjuku-ku, Tokyo 160, Japan.

Copyright (C) 1979 by Pacific Journal of Mathematics Manufactured and first issued in Japan 


\section{Pacific Journal of Mathematics}

\section{Vol. 82 , No. 2 \\ February, 1979}

Krishnaswami Alladi and Paul Erdős, On the asymptotic behavior of large prime

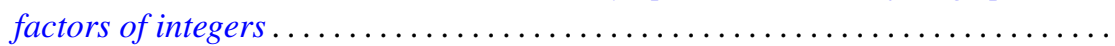

Alfred David Andrew, A remark on generalized Haar systems in $L_{p}$,

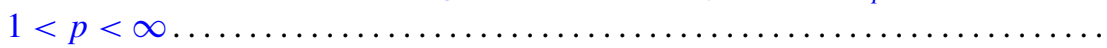

John M. Baker, A note on compact operators which attain their norm . . ........

Jonathan Borwein, Weak local supportability and applications to

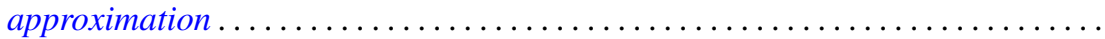

Tae Ho Choe and Young Soo Park, Wallman's type order compactification ........

Susanne Dierolf and Ulrich Schwanengel, Examples of locally compact

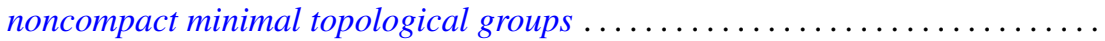

Michael Freedman, A converse to (Milnor-Kervaire theorem) $\times R$ etc. . . . . . . .

George Golightly, Graph-dense linear transformations ..................

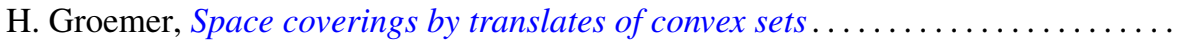

Rolf Wim Henrichs, Weak Frobenius reciprocity and compactness conditions in

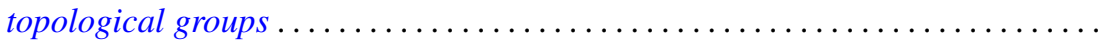

Horst Herrlich and George Edison Strecker, Semi-universal maps and universal

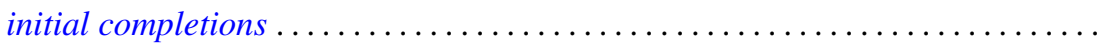

Sigmund Nyrop Hudson, On the topology and geometry of arcwise connected,

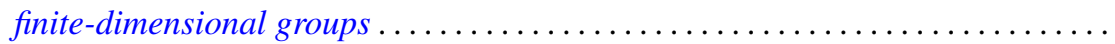

K. John and Václav E. Zizler, On extension of rotund norms. II .............

Russell Allan Johnson, Existence of a strong lifting commuting group of transformations. II.

Bjarni Jónsson and Ivan Rival, Lattice varieties covering the smallest nonmodular variety

Grigori Abramovich Kolesnik, On the order of Dirichlet L-functions .

Robert Allen Liebler and Jay Edward Yellen, In search of nonsolvable groups of

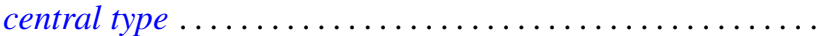

Wilfrido Martínez T. and Adalberto Garcia-Maynez Cervantes, Unicoherent plane Peano sets are $\sigma$-unicoherent ...

M. A. McKiernan, General Pexider equations. I. Existence of injective

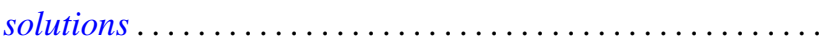

M. A. McKiernan, General Pexider equations. II. An application of the theory of webs.

Jan K. Pachl, Measures as functionals on uniformly continuous functions . .

Lee Albert Rubel, Convolution cut-down in some radical convolution algebras ...

Peter John Slater and William Yslas Vélez, Permutations of the positive integers

with restrictions on the sequence of differences. II . . .

Raymond Earl Smithson, A common fixed point theorem for nested spaces ....

Indulata Sukla, Generalization of a theorem of McFadden .... . . .

Jun-ichi Tanaka, A certain class of total variation measures of analytic measures.

Kalathoor Varadarajan, Modules with supplements .............. 